\title{
SOME CLASSICAL SEQUENCE SPACES AND THEIR TOPOLOGICAL STRUCTURES
}

\author{
Narayan Prasad Pahari \\ Central Department of Mathematics, Tribhuvan University, \\ University Campus, Kirtipur, Kathmandu, Nepal \\ Email Address: nppahari@gmail.com
}

\begin{abstract}
The aim of this paper is to study some of the basic scalar and vector valued sequence spaces. We also study the topological structures of some of the basic sequence spaces when topologized through a norm or a paranorm.
\end{abstract}

Keywords: Function Space, Sequence Space, Paranormed Space, Orlicz Sequence Space.

2010 AMS Subject Classification: Primary - 46A45, Secondary - 46B 15.

\section{Introduction}

Functional Analysis is an abstract branch of Mathematics, that deals with the study of linear spaces endowed with some kinds of limit-related structures like topology, norm, inner product etc. and the operators or functions acting upon these spaces.By a function space we mean a linear space of functions defined on a certain set with respect to pointwise addition and scalar multiplication.

The study of sequence spaces is in fact a special case of the more general study of function spaces if the domain is restricted to the set of natural numbers $\mathbf{N}$.The set $\omega$ of all functions from the natural numbers $\mathbf{N}$ to the field $\mathbf{K}$ of real $\boldsymbol{R}$ or complex numbers $\boldsymbol{C}$, can be turned into a vector space. In other words, let $\omega$ be the set of all (real-or) complex valued sequences $\left\{x_{n}\right\}_{n} \in N$. i.e., $x_{n} \in \boldsymbol{C}$ under the operations of point wise addition and scalar multiplication given by

$$
\begin{aligned}
\qquad\left\{x_{n}\right\}_{n \in N}+\left\{y_{n}\right\}_{n \in N}=\left\{x_{n}+y_{n}\right\}_{n \in N} \\
\text { and } \lambda\left\{x_{n}\right\}_{n \in N}=\left\{\lambda x_{n}\right\}_{n \in N},
\end{aligned}
$$

for every $x_{n}, y_{n} \in \boldsymbol{C}$ and scalar $\lambda$, form a vector space over $\mathbf{C}$. Any subspace $X$ of $\omega$ is then called a sequence space. In other words, a sequence space is a vector space whose elements are infinite scalar sequences of real or complex numbers and is closed under the coordinatewise addition and scalar multiplication. If it is closed under coordinate wise multiplication as well, it is called a sequence algebra. Sequence spaces when equipped with a linear topology form topological vector spaces.

Several workers like Kamthan and Gupta (1980), Maddox (1980), Ruckle (1981), Malkowski and Rakocevic (2004) etc. have made their significant contributions in developing the theory of vector and scalar valued sequence spaces in various directions, when sequences are taken from a Banach space or from a locally convex space. Literatures concerning the theory of sequence space can be 
found in any standard text books and monographs of Functional Analysis, for instance we refer a few; Lindenstrauss and Tzafriri (1977), Wilansky( 1978), Kamthan and Gupta (1980), Rao and Ren (1991) etc.

\section{Topological Structures of Some Basic Sequence Spaces}

We shall study the topological structures of sequence spaces when topologized through a norm or through a paranorm generalize and unify various existing sequence spaces. The concept of paranorm is closely related to linear metric spaces. It is a generalization of that of absolute value of real numbers or modulus of a complex number.

A paranormed space $(X, G)$ is a linear space $X$ together with a function $G: X \rightarrow \boldsymbol{R}_{+}$(called a paranorm on $X$ ) which satisfies the following axioms:

$P N 1: G(\theta)=0$;

PN2: $G(x)=G(-x)$ for all $x \in X$;

$P N 3: G(x+y) \leq G(x)+G(y)$ for all $x, y \in X$; and

PN4: if $\left(\alpha_{n}\right)$ be a sequence of scalars with $\alpha_{n} \rightarrow \alpha$ as $n \rightarrow \infty$ and $\left(x_{n}\right)$ be a sequence in $X$ with $G\left(x_{n}-x\right) \rightarrow 0$ as $n \rightarrow \infty$, then $G\left(\alpha_{n} x_{n}-\alpha x\right) \rightarrow 0$ as $\mathrm{n} \rightarrow \infty$ (continuity of scalar multiplication).

A paranormed space $(X, G)$ is said to be complete if $(X, d)$ is complete with metric

$$
d(x, y)=G(x-y) .
$$

Every paranormed space becomes a linear pseudometric space with $d(x, y)=G(x-y)$ which is translation invariant. Thus each paranormed space is a topological linear space. Moreover a pseudometric linear space must be a paranormed space, i.e., a paranorm can be defined on the space which induces an equivalent pseudometric.

The studies on paranormed sequence spaces were initiated by Nakano and Simons at the initial stage. Later on it was further studied by Maddox [1969] and many others. Bhardwaj and Bala [2007], Khan [2008], and many others studied paranormed sequence spaces using Orlicz function.

We shall discuss the topological structures of some of the important classes of the basic sequence spaces when topologized through a norm or through a paranorm, which in fact, generalize and unify various existing sequence spaces. Among them, sequence spaces $c_{0}(p), c(p), \ell_{\infty}(p)$ appear in the work of Lascarides and Maddox (1971) and others while $c_{0}(X), c(X), \ell_{\infty}(X), \ell_{p}(X)$ are used by Leonard (1976),Maddox (1980) and others. Let $x=\left(x_{k}\right)=\left(x_{k}\right)_{k=1}^{\infty}$ be the sequences and $\omega$ denote the class of all sequences $x=\left(x_{k}\right), k \geq 1$, over the field $\mathbf{C}$ of complex numbers. Let $p=\left(p_{k}\right)$ be any sequence of strictly positive real numbers (bounded in general) and $\lambda=\left(\lambda_{k}\right)$ be any sequence of non zero complex numbers. Here we deal with the topological structures of the following sequence spaces.

\section{A. The spaces $c(p), c_{0}(p), c$ and $c_{0}$.}

With $\left\{p_{k}\right\}$ as above, define

$$
c_{0}(p)=\left\{x=\left\{x_{k}\right\}:\left|x_{k}\right|^{p_{k}} \rightarrow 0 \text { as } k \rightarrow \infty\right\} ; \text { and }
$$




$$
c(p)=\left\{x=\left\{x_{k}\right\}:\left|x_{k}-l\right|^{p_{k}} \rightarrow 0 \text { as } k \rightarrow \infty \text { for some } l \in \boldsymbol{C}\right\} .
$$

$c(p)$ and $c_{0}(p)$ form metric spaces with the metric

$$
d(x, y)=\sup _{k}\left|x_{k}-y_{k}\right|^{p_{k / M}} \text {, where } M=\max \left\{1, \sup p_{k}\right\} .
$$

Note that the set $c(p)$ and $c_{0}(p)$ are complete with its metric topology. But it is not normed space. In fact, the set $c(p)$ forms a paranormed space with paranorm

$$
\|x\|=\sup _{k}\left|x_{k}\right|^{p_{k / M}} \text { if and only if inf } p_{k}>0 .
$$

The set $c_{0}(p)$ is a linear metric space paranormed by

$$
\|x\|=\sup _{k}\left|x_{k}\right|^{p_{k / M}} \text {, where } M=\max \left\{1, \sup p_{k}\right\} .
$$

If $p_{k}=p$ for all $k$, then we write $c$ and $c_{0}$ for $c(p)$ and $c_{0}(p)$ respectively. $c$ and $c_{0}$ are respectively the sets of all convergent sequences and null sequences. Note that $c$ and $c_{0}$ are metric spaces as well as Banach spaces with the metric and norm respectively given by

$$
d(x, y)=\sup _{k}\left|x_{k}-y_{k}\right| \text { and }\|\boldsymbol{x}\|=\sup _{k}\left|x_{k}\right| .
$$

\section{B. The spaces $l_{\infty}(p)$ and $l_{\infty}$.}

Let $\left\{p_{k}\right\}$ be as in the above. We define

$$
l_{\infty}(p)=\left\{x=\left\{x_{k}\right\}: \underset{k}{\sup }\left|x_{k}\right|^{p_{k}<\infty}\right\} .
$$

Note that $l_{\infty}(p)$ is complete metric space with its metric topology with the metric

$$
d(x, y)=\sup _{k}\left|x_{k}-y_{k}\right|^{p_{k / M}} \text {, where } M=\max \left\{1, \sup p_{k}\right\} .
$$

But it is not normed space. In fact, the set $l_{\infty}(p)$ is paranormed space with paranorm

$$
\|x\|=\sup \left|x_{k}\right|^{p_{k / M}} \text { if and only if inf } p_{k}>0 .
$$

If $p_{k}=p$ for all $k$, then we write $l_{\infty}$ for $l_{\infty}(p)$. $l_{\infty}$ is the set of all bounded sequences $x=\left\{x_{k}\right\}$ of real (or complex) numbers and forms a metric space and Banach space with the natural metric and norm respectively given by

$$
d(x, y)=\sup _{k}\left|x_{k}-y_{k}\right| \text { and }\|x\|=\sup _{k}\left|x_{k}\right| .
$$

\section{The spaces $\ell(p)$ and $\ell_{p}$.}

Since $\left\{p_{k}\right\}$ be a bounded sequence of strictly positive real numbers, so that $0<p_{k} \leq \sup p_{k}<\infty$. We define

$$
l(p)=\left\{x=\left\{x_{k}\right\}: \sum_{k=1}^{\infty}\left|x_{k}\right|^{p_{k}}<\infty\right\} .
$$

Then, $l(p)$ becomes a metric space with metric 


$$
d(x, y)=\left(\sum_{k=1}^{\infty}\left|x_{k}-y_{k}\right|^{p_{k}}\right)^{1 / M} \text {, where } M=\max \left\{1, \operatorname{Sup} p_{k}\right\} .
$$

The space $l(p)$ is complete metric space in its metric topology but it is not a normed space.

If fact, the set $l(p)$ is paranormed by

$$
\|x\|=\left(\sum_{k=1}^{\infty}\left|x_{k}\right|^{p_{k}}\right)^{1 / M} .
$$

In particular, if $p_{k}=p$ for all $k$, then we write $l_{p}$ for $l(p)$, which consists of the $p$-power summable sequences with $p$-norm.

Observe that if $1 \leq p<\infty, l_{p}$ forms a metric space and Banach space with the metric and norm respectively given by

$$
d(x, y)=\left(\sum_{k=1}^{\infty}\left|x_{k}-y_{k}\right|^{p}\right)^{1 / p} \text { and }\|x\|=\left(\sum_{k=1}^{\infty}\left|x_{k}\right|^{p}\right)^{1 / p} .
$$

Similarly for $0<p<1, l_{p}$ forms a metric space and complete $p$-normed space with the metric and $p$ norm respectively given by

$$
d(x, y)=\sum_{k=1}^{\infty}\left|x_{k}-y_{k}\right|^{p} \text { and }\|x\|=\sum_{k=1}^{\infty}\left|x_{k}\right|^{p} .
$$

\section{Orlicz Sequence Space $\ell_{\Phi}$}

The study of Orlicz sequence spaces was initiated to study Banach space theory. An Orlicz function is a function $\Phi:[0, \infty) \rightarrow[0, \infty)$ which is continuous, non decreasing and convex with

$$
\Phi(0)=0, \Phi(u)>0 \text { for } u>0 \text {, and } \Phi(u) \rightarrow \infty \text { as } u \rightarrow \infty .
$$

Lindenstrauss and Tzafriri (1977) used Orlicz function to construct the sequence space

$$
\ell_{\Phi}=\left\{x=\left(x_{k}\right) \in \omega: \sum_{k=1}^{\infty} \Phi\left(\frac{\left|x_{k}\right|}{\rho}\right)<\infty \text { for some } \rho>0\right\}
$$

of scalars, which forms a Banach space with Luxemburg norm defined by

$$
\|x\|_{\Phi}=\inf \left\{\rho>0: \sum_{k=1}^{\infty} \Phi\left(\frac{\left|x_{k}\right|}{\rho}\right) \leq 1\right\} .
$$

The space $\ell_{\Phi}$ is called an Orlicz sequence space and is closely related to the space $\ell_{p}$ with $\Phi(x)=x^{p},(1 \leq p<\infty)$. They have very rich topological and geometrical properties that do not occur in ordinary $\ell_{p}$ spaces.

Bhardwaj and Bala (2007), Khan (2008), Kolk (2011),Pahari and Srivastava (2011),(2012), and many others, have been introduced and studied various sequence spaces using Orlicz function as a generalization of well known sequence spaces . 


\section{E. Other Sequence Spaces}

We shall denote $e$ and $e^{(n)}(n=1,2, \ldots)$ for the sequences such that $e_{k}=1$ for $k=1,2, \ldots$ and

$$
e_{k}^{(n)}= \begin{cases}1, & (\text { for } k=n) \\ 0, & (\text { for } k \neq n) .\end{cases}
$$

Let $m$ be any nonnegative integer, we denote the $m$-section of a sequence $x=\left\{x_{k}\right\}$ by $x^{[m]}$, i.e.

$$
x^{[m]}=\sum_{k=1}^{\infty} \cdot x_{k} e^{(k)}
$$

Further $C S, l_{1}$ and $B S$ denote for the sets of all convergent, absolutely convergent and bounded series as described below:

The space of bounded series $B S$ is the space of sequences $X$ for which $\sup \left|\begin{array}{cc}n \\ n\end{array}\right| \begin{array}{cc}\sum_{k} & x_{k}\end{array} \mid<\infty$.

The space $B S$, when equipped with the norm

$$
\|x\|_{B S}=\sup _{n}\left|\begin{array}{cc}
n \\
k=1
\end{array}\right|
$$

forms a Banach space isometrically isomorphic to $l^{\infty}$, via the linear mapping

$$
\left(x_{n}\right)_{n \in N} \rightarrow\left(\begin{array}{cc}
n \\
\sum_{k=1} & x_{k}
\end{array}\right)_{n \in N} .
$$

A sequence space $X$ with a linear topology is called a $\boldsymbol{K}$-space provided each of the maps $\quad p_{k}: X \rightarrow$ $\boldsymbol{C}$ defined by $p_{k}(x)=x_{k}, x \in X$ is continuous for all $k=1,2, \ldots$

A $K$-space $X$ is called an $\boldsymbol{F K}$ - space provided $X$ is a complete linear metric space. A normed $F K$ space is called a $\boldsymbol{B} \boldsymbol{K}$-space.

Further, $l_{l}$ is a $B K$ space with respect to the norm

$$
\|x\|_{1}=\sum_{k=1}^{\infty}\left|x_{k}\right|
$$

$c_{0}, c$ and $l_{\infty}$ are $B K$ spaces with respect to the norm

$$
\|x\|_{\infty}=\underset{k}{\sup }\left|x_{k}\right|
$$

and $c s$ is a $B K$ space with respect to the norm

$$
\|x\|_{C S}=\underset{n}{\sup }\left|\sum_{k=1}^{n} \cdot x_{k}\right| \text {,see, Malkowski (1999). }
$$


A $F K$ space $X \supset \phi$ is said to have $\boldsymbol{A K}$ - space if every sequence $x=\left\{x_{k}\right\}_{k=1}^{\infty}$ in $X$ has a unique representation of the form $x=\sum_{k=1}^{\infty} x_{k} e^{(k)}$, i.e. $x^{[m]} \rightarrow x($ as $m \rightarrow \infty)$.For examples, the spaces $l_{l}, c_{0}$ and $C S$ form $A K$ spaces.

A sequence $\left(x^{(k)}\right)_{k=1}^{\infty}$ in a linear metric space $X$ is called a Schauder basis (see, Malkowski, 1999), if for every $x \in X$ there exists a uniquely determined sequence $\left\{\lambda_{k}\right\}_{k=1}^{\infty}$ of scalars such that

$$
x=\sum_{k=1}^{\infty} \lambda_{k} x^{(k)}
$$

A complex sequence $\left\{x_{k}\right\}$ is called an analytic sequence if the sequence $\left\{\left|x_{k}\right|^{1 / k}\right\}$ is bounded and an entire sequence if $\left|x_{k}\right|^{1 / k} \rightarrow 0$ as $k \rightarrow \infty$. The set of all analytic and entire sequences are respectively denoted by $\varpi$ and $\Gamma$, see Rao (1999).

\section{Conclusion}

In this paper, we have characterized the topological structures of some of the basic scalar and vector valued sequence spaces. In fact, these structures can also be used to explore further properties of the generalized sequence spaces by topologizing through a norm or a paranorm. 


\section{References}

1. V. N. Bhardwaj, and I. Bala, "Banach space valued sequence space $\ell_{M}(X, p)$ ", Int. J. of Pure and Appl. Maths”, 41(5),617-626, 2007.

2. P. K. Kamthan, and M. Gupta, "Sequence and series, lecture notes", Marcel Dekker Inc., 1980.

3. V. A. Khan, "On a new sequence space defined by Orlicz functions”, Common. Fac Sci. Univ. Ank-series; 57( 2) , 25-33, 2008.

4. E. Kolk," Topologies in generalized Orlicz sequence spaces”, Filomat, 25(4), 191-211, 2011.

5. Lascarides, C.G. and Maddox, I.J. "A study of certain sequence spaces of Maddox and generalization of a theorem of Iyer”; Pacific. J. Math ; 38, 481-499, 1971.

6. I. E. Leonard, "Banach sequence space"; J. Math Anal. Appl.54(1), 245-265, 1976.

7. J. Lindenstrauss, and L. Tzafriri, "Classical Banach spaces", Springer-Verlag, New York/Berlin, 1977.

8. I. J. Maddox, “Some properties of paranormed sequence spaces”, J. Math. Soc. London. 2(1), 316-322, 1969.

9. E. Malkowski, "On Generalized Matrix Domains; Sequence Spaces and Applications"; Narosa Publishing House, New Delhi, 35-37, 1999.

10. E. Malkowski, and V. Rakocevic, "An introduction into the theory of sequence spaces and measures of non-compactness”, 2004.

11. N.P. Pahari, and J. K. Srivastava,"On Banach space valued paranormed sequence space $\ell_{M}(X$,

$\bar{\lambda}, \bar{p}, L)$ defined by Orlicz function”; South East Asian J.Math. \& Math.Sc.; 10(1), 45-57, 2011.

12. N. P. Pahari, and J. K. Srivastava, "On vector valued paranormed sequence space $c_{0}(X, M, \bar{\lambda}, \bar{p})$ defined by Orlicz function”; J.Rajasthan Acad. of Phy. Sci.; 11(2),11-24, 2012.

13. K. C. Rao, "The Space of Entire Sequences; Sequences Spaces and Application”, Narosa Publishing House, New Delhi, 4-8, 1999.

14. M. M. Rao, and Z. D. Ren, "The Theory of Orlicz Space”, Marcel Dekker Inc., New York, 1991.

15. W. H. Ruckle, “Sequence spaces”, Pitman Advanced Publishing Programme, 1981.

16. S. Simons,"The sequence spaces $l\left(p_{\nu}\right)$ and $m\left(p_{\nu}\right)$ ") ; Proc. London Math. Soc. (3),15, 422-436, 1965.

17. A. Wilansky, "Modern methods in topological vector spaces", McGraw-Hill Book Co. Inc. New York, 1978. 